\title{
In Vitro Activity of Caspofungin Against Fluconazole-Resistant Candida Species Isolated From Clinical Samples in Iran
}

\author{
Hamideh Shekari Ebrahim Abad ${ }^{1}$; Farideh Zaini ${ }^{1, *}$; Parivash Kordbacheh ${ }^{1}$; Mahmoud \\ Mahmoudi ${ }^{2}$; Mahin Safara ${ }^{1}$; Vida Mortezaee ${ }^{1}$ \\ ${ }^{1}$ Department of Parasitology and Mycology, School of Public Health, Institute of Public Health Researches, Tehran University of Medical Sciences, Tehran, IR Iran \\ ${ }^{2}$ Department of Epidemiology and Biostatistics, School of Public Health, Tehran University of Medical Sciences, Tehran, IR Iran \\ ${ }^{*}$ Corresponding author: Farideh Zaini, Department of Parasitology and Mycology, School of Public Health, Institute of Public Health Researches, Tehran University of Medical \\ Sciences, P. O. BOX: 6446/14155, Tehran, IR Iran. Tel:+98-2188951391, Fax:+98-2166462267, E-mail: Fzaini@sina.tums.ac.ir
}

Received: February 19, 2014; Revised: June 16, 2014; Accepted: July 20, 2014

\begin{abstract}
Background: Candida spp. is the most common organisms involved in fungal infections in the high risk patients. It causes the greatest number of invasive candidiasis. Fluconazole is effective in treating mucosal candidiasis. However, resistance to fluconazole and other azoles antifungal drugs is an important clinical problem to treat candidiasis. Caspofungin is more effective against Candida species such as some azoles-resistant isolates.

Objectives: The current study aimed to investigate the susceptibilities of clinical fluconazole-resistant and fluconazole - susceptible dosedependent Candida species to caspofungin.

Materials and Methods: In the Minimum Inhibitory Concentration(MIC) test, 207 Candida species and other yeasts isolated from Iranian patients (each isolated from a high-risk patient) were evaluated. The yeasts were differentiated by standard mycological methods, CHROM agar Candida, and verified by API20C.AUX. In vitro susceptibilities were determined using Broth Micro Dilution(BMD) method described in the Clinical Laboratory Standards Institute M27-A3. MICs were noted after 24 and 48 hours of incubation.

Results: The most frequently isolated species were Candida albicans (52.2\%), C. glabrata (24.6\%), followed by C. tropicalis (7.7\%) and C. krusei (3.4\%). MICs of caspofungin against $87 \%$ of C. albicans and $90 \%$ of C. glabrata and C. tropicalis isolates were $2 \mu \mathrm{g} / \mathrm{mL}$ and for C. krusei were $4 \mu \mathrm{g} / \mathrm{mL}$, respectively. The results revealed that only 20 out of 207 isolates (9.7\%) were non-sensitive to caspofungin. Caspofungin nonsusceptible isolates were isolated from the patients with cancer, diabetes and AIDS; and not in the species isolated from patients with other underlying diseases.
\end{abstract}

Conclusions: Caspofungin appears more effective in vitro against Iranian fluconazole-resistant Candida isolates and some other yeasts.

Keywords: Fluconazole; Caspofungin; Candida albicans

\section{Background}

The most commonly used classes of antifungal agents to treat Candida infections are the azoles, polyenes, and echinocandins (1). Many factors including the excessive use of broad-spectrum antimicrobial agents, aggressive anticancer therapy and the AIDS epidemic increase the incidence of candidiasis $(2,3)$. The most common organisms involved in fungal infections in the high risk patients is Candida spp. that cause the greatest number of invasive candidiasis (4). Caspofungin is an echinocandins antifungal exhibiting significant in vitro activity against the Candida spp. (5). Fluconazole is effective in treating mucosal candidiasis. However, resistance to fluconazole and other azoles antifungal drugs is an important clinical problem to treat candidiasis (6). Caspofungin is more effective against Candida species, including some azoles-resistant isolates $(6,7)$. Caspofungin has excellent antifungal activity against many non-albicans Candida species, particularly Candida glabrata isolates expressing resistant to fluconazole (8-12).
Antifungal drug resistance is rapidly changing a major problem; especially with the immunocompromised patients $(13,14)$. Considering the increased fluconazoleresistance Candida spp. isolates the need arises for antifungal susceptibility testing $(14,15)$. In Iran, there is not enough information about the effect of caspofungin against fluconazole-resistance Candida spp. isolates in high-risk patients including the ones with diabetes, immunodeficiency, organs and bone marrow transplantations, cancers, immunosuppressive drugs users etc.

\section{Objectives}

The current study aimed to investigate the susceptibilities of clinical fluconazole-resistant and fluconazole-susceptible dose-dependent Candida species to caspofungin.

\section{Materials and Methods}

The study protocol conforms to the ethical guidelines

Copyright (C) 2015, Ahvaz Jundishapur University of Medical Sciences. This is an open-access article distributed under the terms of the Creative Commons Attribution-NonCommercial 4.0 International License (http://creativecommons.org/licenses/by-nc/4.0/) which permits copy and redistribute the material just in noncommercial usages, provided the original work is properly cited. 
of the 1975 Helsinki Declaration as reflected in a priori approval by the Human Research Committee Institution and informed consent letter was obtained from each patient participated in the study (2).

\subsection{Organisms}

Two-hundred and seven Candida species including C. albicans, C. parapsilosis, C. tropicalis, C. glabrata, C. krusei, C. guilliermondi, C. kefyr etc. isolated from high-risk patients including the ones with HIV-positive, cancer, diabetes and etc. and identified by standard methods (cornmeal for blastoconidia, germ-tube formation, pseudohyphae, and true hyphae and growth on Chrome Candida agar) and the API 20 C system (16) were examined by fluconazole in pretest; In addition, 118 ATCC 22019 and C. krusei ATCC 6285 which are standard species of $C$. parapsilosis included in each run of susceptibility tests for quality control.

\subsection{Antifungal Drugs}

To prepare caspofungin stocks, caspofungin (Merck and Co., Inc., NJ, USA) by broth micro dilution assay was used, according to the Clinical and Laboratory Standard Institute (CLSI) (16).

\subsection{Preparing Caspofungin Dilution}

Caspofungin stock solution, according to NCCLS micro dilution method (17), was prepared by adding $0.0256 \mathrm{~g}$ caspofungin to a falcon tube including $10 \mathrm{~mL}$ distilled water and then the tube was incubated in $-70^{\circ} \mathrm{C}$ for further applications. RPMI 1640 (sigma Aldrich) in association with L-glutamine (without sodium bicarbonate) and buffered ( $\mathrm{pH}=7.0$ ) with MOPS was used as susceptibility test and serial two-fold dilutions.

\subsection{Culturing the Isolates}

Initially, all isolates including 108 (52.2\%) C. albicans, 51 (24.6\%) C. glabrata, 16 (7.7\%) C. tropicalis, 7 (3.4\%) C. krusei, 4 (1.9\%) C. dubliniensis, other Candida spp. 11 (5.3\%) (one C. parapsilosis, two C. guilliermondi, three C. kefyer, three $C$. femata, one C. incanspiqua and one C.ciferi) and unknown yeasts 10 (4.8\%) were cultured on Sabouraud dextrose agar (Merck, Germany) and incubated at $37^{\circ} \mathrm{C}$ for 24 hours.

\subsection{Preparing Yeast Suspensions}

Yeast inoculums was prepared by picking two to three colonies of $>1 \mathrm{~mm}$ diameter from an overnight culture of Candida species, growing on Sabouraud dextrose agar at $35^{\circ} \mathrm{C}$, and suspending them in $2 \mathrm{~mL}$ of $0.85 \%$ normal saline. The resulting suspension was vortexed for $15 \mathrm{sec}-$ onds and this procedure yielded a yeast stock suspension of $1 \times 10^{6}-5 \times 10^{6}$ cells/mL. A working suspension was made by a 1:100 dilution followed by a 1:20 dilution of the stock suspension with RPMI 1640 broth medium, which results in $0.5-2.5 \times 10^{3}$ cells $/ \mathrm{mL}$. The cell density was adjusted to $0.5-2.5 \times 10^{3}$ cells $/ \mathrm{mL}$.

\subsection{Candida spp. Isolates Sensitivity to Caspofungin}

Micro dilution plates were set up according to the NCCLS (CLSI) M27-A3 guidelines. Thermo Scientific ${ }^{\mathrm{TM}}$ Nunc $^{\mathrm{TM}}$ MicroWell ${ }^{\mathrm{TM}}$ with 96-well flat-bottomed micro dilution panels is ideal for microscopic and optical measurements. Each micro plate had two drug free growth controls, one with the media alone (growth control) and the other with media containing an equivalent amount of solvent used to dissolve the drug (solvent control).

\subsection{Minimum Inhibitory Concentration}

Plates were incubated at $35^{\circ} \mathrm{C}$ and MICs were read visually after 48 hours using mirror. Rates of resistance were determined according to the MIC breakpoints proposed by NCCLS (CLSI) M27-A3. Caspofungin, an MIC of $>2 \mu \mathrm{g} /$ $\mathrm{mL}$, was used to identify caspofungin-non-susceptible Candida spp. (18). Fluconazole with the MICs of $>64 \mu \mathrm{g} /$ $\mathrm{mL}$, and 16 - $32 \mu \mathrm{g} / \mathrm{mL}$ were used to identify resistance, and susceptible dose-dependence against fluconazole, respectively (19). The growth level in each well was compared to that of the positive control. Antifungal activity was expressed as the MIC of each drug against the isolate. The following resistance breakpoints were used according to CLSI guidelines (17). MIC rates of caspofungin against C. parapsilosis ATCC 22019 were 1 and $0.0625 \mu \mathrm{g}$ / $\mathrm{mL}$, and against C. krusei ATCC 6258 were 0.5 and $0.5 \mu \mathrm{g} /$ $\mathrm{mL}$, respectively. MIC50 and MIC90 were also calculated. Data were analyzed by SPSS version 11.5.

\begin{tabular}{|c|c|c|c|}
\hline & $\begin{array}{l}\text { Fluconazole-Resistant } \\
\text { Isolates MIC } \geq 64\end{array}$ & $\begin{array}{c}\text { Caspofungin-Non Susceptible Isolates } \\
(\mathrm{MIC}>2) \text { in Fluconazole-Resistant Isolates }\end{array}$ & $\begin{array}{c}\text { Caspofungin-Susceptible Isolates } \\
(\mathrm{MIC} \leq 2) \text { in Fluconazole-Resistant Isolates }\end{array}$ \\
\hline C.albicans (108) & $8(7.4)$ & $1(12.5)$ & $7(87.5)$ \\
\hline C.glabrata (51) & $15(29.4)$ & $1(6.6)$ & $14(93.4)$ \\
\hline C. tropical (16) & $3(18.7)$ & 0 & $3(100)$ \\
\hline C. krusei (7) & $5(71.4)$ & $1(20)$ & $4(80)$ \\
\hline C. dubliniensis (4) & 0 & 0 & 0 \\
\hline Other Candia spp.(11) & 0 & 0 & 0 \\
\hline Unknown yeasts (10) & $2(20)$ & 0 & $2(100)$ \\
\hline Total (207) & $33(16)$ & $3(9)$ & $30(91)$ \\
\hline
\end{tabular}




\section{Results}

The most abundant species isolated from high-risk patients with cancer, AIDS and diabetes were C. albicans (52.2\%), C.glabrata (24.6\%), C. tropicalis (7.7\%) and C.krusei (3.4\%), respectively. The results revealed that 30 (91\%) out of 33 fluconazole-resistant isolates of Candida spp. and other yeasts were susceptible to caspofungin. Only 3 (9\%) out of 33 fluconazole-resistant isolates of Candida spp. were non-susceptible to caspofungin. The current study showed that in vitro caspofungin was more active against Iranian fluconazole-resistant Candida spp. and some other yeasts isolated from clinical samples in Iran $(\mathrm{P}<0.001)$.

\section{Discussion}

Despite improvements in medical products, some health problems still remain unchanged $(20,21)$. Furthermore, innate or acquired antifungal resistance may pose a serious problem to antifungal treatment $(20,21)$. Antifungal resistance among invasive isolates of Candida is not common; therefore, it is still a concern, particularly for C. glabrata and C. krusei $(22,23)$, both of which are used to show intrinsic (C. krusei) or acquired (C. glabrata) resistance against fluconazole (24). Caspofungin is a fungicidal echinocandins and is active against many species of Candida (24-27). Caspofungin and other echinocandins exhibit potent activity against fluconazole-resistant Candida spp. (24, 26, 28-30). Pfaller et al. determined the in vitro activity of caspofungin against 351 fluconazoleresistant Candida isolates (24), and reported that 99\% were susceptible to caspofungin at the MIC of $2 \mathrm{~g} / \mathrm{mL}$ (24). Bachmann et al. reported that caspofungin was equally active against fluconazole-susceptible and fluconazoleresistant isolates (6). Lyon et al. evaluated the susceptibility of 5,821 isolates of Candida spp. They reported that $C$. albicans, C. parapsilosis, C. tropicalis, and C. lusitaniae were quite susceptible to fluconazole and C. glabrata was less susceptible to fluconazole (31).

Caspofungin exhibited (99.8\%) significant activity against all species of Candida Lemos et al. reported 157 fluconazole-resistant Candida isolates out of 3959 Candida species isolated from clinical samples (2). The current study showed that caspofungin was active against the fluconazole- resistant Candida isolates (2). Silver et al. evaluated in vitro susceptibility of 80 C. glabrata species isolated from clinical samples to caspofungin (L). Their results showed that caspofungin had significant activity against fluconazole-resistant isolates, isolates susceptible and dose-dependent susceptible to fluconazole (32). Evaluated the activity of caspofungin against 3,959 isolates of Candida spp., and reported that a total of 157 isolates were resistant to fluconazole. Caspofungin showed the same activity against fluconazole-resistant isolates that it showed against isolates susceptible and dosedependently susceptible to fluconazole (33). The current study determined in vitro activity of caspofungin against
Iranian fluconazole-resistant Candida spp. isolated from clinical samples in Iran. Results showed that 30 (91\%) out of 33 isolates were susceptible to caspofungin and 95\% of the fluconazole-resistant, susceptible, and the growth of dose dependent Candida spp. isolates were inhibited by caspofungin; 7 (87.5\%) out of 8 C. albicans, 14 (93.4\%) out of 15 C. glabrata and 4 (80\%) out of 5 C. krusei fluconazoleresistant isolates were inhibited by MIC $\leq 2$ of caspofungin; the growth of $100 \%$ of C. tropicalis were inhibited by caspofungin. These findings confirm and extend the ones previously reported regarding significant activity of caspofungin $(1,2,6,24,31-33)$.

Ortiz de la Tabla-Ducasse et al. in their in vitro study reported that caspofungin was very active against a variety of fluconazole-resistant Candida strains isolated from clinical cohort of HIV-infected patients. The MIC50 and MIC ranges of caspofungin against $C$. albicans were slightly higher than those of C. glabrata, which confirm the results of the current study (34). Posteraro et al. reported that their results represent further evidence for the excellent antifungal potency of caspofungin, particularly against $C$. glabrata isolates, expressing cross-resistance to azoles that confirms the results of the current study (8). The current in vitro study showed that caspofungin appears to be more effective against fluconazole-resistant Candida species and some other yeasts isolated from clinical samples in Iran.

\section{Authors' Contributions}

Conception, study design/provision, patients/sample collection and data assembling: Farideh Zaini, Mahin Safara, Parivash Kordbacheh, Hamideh Shekari Ebrahim Abad, Vida Mortezaee; data analysis and interpretation: Mahmoud Mahmoudi; Manuscript writing: Hamideh Shekari Ebrahim, Vida Mortezaee; Final Approval: Farideh Zaini.

\section{Funding/Support}

This study was funded and supported by Tehran University of Medical Sciences.

\section{References}

1. Hoehamer CF, Cummings ED, Hilliard GM, Rogers PD. Changes in the proteome of Candida albicans in response to azole, polyene, and echinocandin antifungal agents. Antimicrob Agents Chemother. 2010;54(5):1655-64.

2. Lemos J, Costa CR, Araújo C, Souza LKH, Silva M. Susceptibility testing of Candida albicans isolated from oropharyngeal mucosa of HIV+ patients to fluconazole, amphotericin B and Caspofungin: killing kinetics of caspofungin and amphotericin B against fluconazole resistant and susceptible isolates. Brazilian J Microbiol. 2009;40(1):163-9.

3. Chattopadhyay A, Caplan DJ, Slade GD, Shugars DC, Tien HC, Patton LL. Risk indicators for oral candidiasis and oral hairy leukoplakia in HIV-infected adults. Community Dent Oral Epidemiol. 2005;33(1):35-44.

4. Garnacho-Montero J, Diaz-Martin A, Ruiz-Perez De Piappon M, Garcia-Cabrera E. [Invasive fungal infection in critically ill patients]. Enferm Infecc Microbiol Clin. 2012;30(6):338-43.

5. Majoros L, Kardos G, Szabo B, Sipiczki M. Caspofungin suscepti- 
bility testing of Candida inconspicua: correlation of different methods with the minimal fungicidal concentration. Antimicrob Agents Chemother. 2005;49(8):3486-8.

6. Bachmann SP, Patterson TF, Lopez-Ribot JL. In vitro activity of caspofungin (MK-0991) against Candida albicans clinical isolates displaying different mechanisms of azole resistance. J Clin Microbiol. 2002;40(6):2228-30.

7. Barchiesi F, Schimizzi AM, Fothergill AW, Scalise G, Rinaldi MG. In vitro activity of the new echinocandin antifungal, MK-0991, against common and uncommon clinical isolates of Candida species. Eur J Clin Microbiol Infect Dis. 1999;18(4):302-4.

8. Posteraro B, Sanguinetti M, Fiori B, La Sorda M, Spanu T, Sanglard D, et al. Caspofungin activity against clinical isolates of azole cross-resistant Candida glabrata overexpressing efflux pump genes. J Antimicrob Chemother. 2006;58(2):458-61.

9. Nagappan V, Boikov D, Vazquez JA. Assessment of the in vitro kinetic activity of caspofungin against Candida glabrata. Antimicrob Agents Chemother. 2010;54(1):522-5.

10. Bartizal C, Odds FC. Influences of methodological variables on susceptibility testing of caspofungin against Candida species and Aspergillus fumigatus. Antimicrob Agents Chemother. 2003;47(7):2100-7.

11. Pfaller MA, Messer SA, Boyken L, Rice C, Tendolkar S, Hollis RJ, et al. Caspofungin activity against clinical isolates of fluconazoleresistant Candida. J Clin Microbiol. 2003;41(12):5729-31.

12. Rex JH, Rinaldi MG, Pfaller MA. Resistance of Candida species to fluconazole. Antimicrob Agents Chemother.1995;39(1):1-8.

13. Jabra-Rizk MA, Falkler WA, Meiller TF. Fungal biofilms and drug resistance. Emerg Infect Dis. 2004;10(1):14-9.

14. Enwuru CA, Ogunledun A, Idika N, Enwuru NV, Ogbonna F, Aniedobe $\mathrm{M}$, et al. Fluconazole resistant opportunistic oro-pharyngeal Candida and non-Candida yeast-like isolates from HIV infected patients attending ARV clinics in Lagos, Nigeria. Afr Health Sci. 2008;8(3):142-8.

15. Lewis RE, Klepser ME, Pfaller MA. Update on clinical antifungal susceptibility testing for Candida species. Pharmacotherapy. 1998;18(3):509-15.

16. Badiee P, Alborzi A, Davarpanah MA, Shakiba E. Distributions and antifungal susceptibility of Candida species from mucosal sites in HIV positive patients. Arch Iran Med. 2010;13(4):282-7.

17. Clinical and Laboratory Standards Institute.. Reference Method for Broth Dilution Antifungal Susceptibility Testing of Yeasts. 3 edUSA: CLSI, Wayne; 2008.

18. Denning DW. Echinocandins: a new class of antifungal. J Antimicrob Chemother. 2002;49(6):889-91.

19. National committee for clinical laboratory standards (NCCLSCLSI).. reference method for broth dilution antifungal susceptibility testing atyeasts: approved standard:; 2002.

20. Fera MT, La Camera E, De Sarro A. New triazoles and echinocandins: mode of action, in vitro activity and mechanisms of resistance. Expert Rev Anti Infect Ther. 2009;7(8):981-98.

21. Mortezaee V, Zaini F, Kordbacheh P, Mahmoudi M, Safara M, Shek ari $\mathrm{H}$. In vitro Susceptibilities of Iranian Clinical non-albicans
Candida species to Caspofungin and Ketoconazole. J Basic Appl Sci Res. 2012;2(2):1608-14.

22. Pfaller MA, Messer SA, Boyken L, Tendolkar S, Hollis RJ, Diekema DJ. Variation in susceptibility of bloodstream isolates of Candida glabrata to fluconazole according to patient age and geographic location. J Clin Microbiol. 2003;41(5):2176-9.

23. Rex JH, Walsh TJ, Sobel JD, Filler SG, Pappas PG, Dismukes $W E$, et al. Practice guidelines for the treatment of candidiasis. Infectious Diseases Society of America. Clin Infect Dis. 2000;30(4):662-78.

24. Pfaller MA, Diekema DJ, Messer SA, Boyken L, Hollis RJ, Jones RN, et al. In vitro activities of voriconazole, posaconazole, and four licensed systemic antifungal agents against Candida species infrequently isolated from blood. J Clin Microbiol. 2003;41(1):78-83.

25. Bartizal K, Gill CJ, Abruzzo GK, Flattery AM, Kong L, Scott PM, et al. In vitro preclinical evaluation studies with the echinocandin antifungal MK-0991 (L-743,872). Antimicrob Agents Chemother. 1997;41(11):2326-32.

26. Ernst EJ, Klepser ME, Ernst ME, Messer SA, Pfaller MA. In vitro pharmacodynamic properties of MK-0991 determined by time-kill methods. Diagn Microbiol Infect Dis. 1999;33(2):75-80.

27. Ernst EJ, Klepser ME, Pfaller MA. Postantifungal effects of echinocandin, azole, and polyene antifungal agents against Candida albicans and Cryptococcus neoformans. Antimicrob Agents Chemother. 2000;44(4):1108-11.

28. Ernst ME, Klepser ME, Wolfe EJ, Pfaller MA. Antifungal dynamics of LY 303366, an investigational echinocandin B analog, against Candida ssp. Diagn Microbiol Infect Dis. 1996;26(3-4):125-31.

29. Martinez-Suarez JV, Rodriguez-Tudela JL. In vitro activities of semisynthetic pneumocandin L-733,560 against fluconazoleresistant and -susceptible Candida albicans isolates. Antimicrob Agents Chemother. 1996;40(5):1277-9.

30. Nelson PW, Lozano-Chiu M, Rex JH. In vitrogrowth-inhibitory activity of pneumocandins L-733,560 and L-743,872 against putatively amphotericin B- and fluconazole-resistantCandidaisolates: influence of assay conditions. Med Mycol. 1997;35(4):285-7.

31. Lyon GM, Karatela S, Sunay S, Adiri Y, Candida Surveillance Study I. Antifungal susceptibility testing of Candida isolates from the Candida surveillance study. J Clin Microbiol. 2010;48(4):1270-5.

32. Silver PM, Oliver BG, White TC. Characterization of caspofungin susceptibilities by broth and agar in Candida albicans clinical isolates with characterized mechanisms of azole resistance. Med Mycol. 2008;46(3):231-9.

33. Pfaller MA, Diekema DJ, Messer SA, Hollis RJ, Jones RN. In vitro activities of caspofungin compared with those of fluconazole and itraconazole against 3,959 clinical isolates of Candida spp. including 157 fluconazole-resistant isolates. Antimicrob Agents Chemother. 2003;47(3):1068-71.

34. Ortiz de la Tabla-Ducasse V, Masia-Canuto M, Martin-Gonzalez C, Gutierrez-Rodero F. [In vitro activity of caspofungin against fluconazole-resistant Candida isolates from patients with HIV infection]. Enferm Infecc Microbiol Clin. 2004;22(6):328-31. 